\title{
Overcoming the immune privilege of $B$ cell follicles to cure HIV-I infection
}

\section{Editorial}

Over 30 million individuals are infected with HIV-1 world-wide. The disease produces great human suffering and extracts an enormous financial toll. Although antiretroviral drugs have produced substantial reductions in HIV-1 related morbidity and mortality, they are costly, associated with side effects, and ineffective in the presence of drug resistant virus. Despite intensive efforts, both a protective vaccine and a cure for HIV-1 remain elusive.

Ongoing HIV-1 replication despite host immune responses to the virus is an enigma and poses a major barrier to the development of a vaccine or a cure. HIV-1 replication occurs primarily in CD4+T cells within secondary lymphoid tissues ${ }^{1,2}$ and results in a progressive decline of CD4+T cells, immunodeficiency, and ultimately death in most untreated individuals. Within secondary lymphoid tissues, HIV1 replication is highly concentrated in follicular CD4+T cells, ${ }^{3-10}$ which are 30-40 times more likely to be productively infected than extra follicular CD4+T cells during chronic HIV-1 infection. ${ }^{3}$ Consequently, follicular CD4+T cells account for approximately $70 \%$ of HIV-1 producing cells in chronic disease prior to AIDS. ${ }^{3,4}$ Follicular dendritic cells (FDC) located in germinal centers of follicles, bind HIV-antibody complexes, and these complexes readily infect CD4+T cells in vitro, ${ }^{11}$ thus providing an explanation for the high rate of infection of follicular CD4+T cells. Nevertheless, it is unclear why HIV-1-specific CD8+T cells are unable to fully suppress HIV-1 replication in follicular CD4+T cells, as they are able to kill productively infected cells in vitro within minutes after they initiate transcription of virus and long before infectious virions are produced.

Multiple lines of evidence indicate that HIV-1-specific CD8+T cells play a pivotal role in controlling virus replication. Development of HIV-1-specific CD8+T cells during acute infection coincides with declines in viremia ${ }^{12-14}$ suggesting that CTL are critical determinants of the initial control of virus replication. In the SIV-infected rhesus macaque model of HIV-1, temporary removal of CD8+T cells leads to increased viremia, and the subsequent return of CD $8+T$ cells correlates with decreased viremia, ${ }^{15,16}$ further implicating CD8+T cells in viral control. Levels of polyfunctional CD8+T cells inversely correlate with virus set point ${ }^{17}$ and polyfunctional $\mathrm{CD} 8+\mathrm{T}$ cells are maintained in HIV-1 infected nonprogressors. ${ }^{18}$ Furthermore, there is a strong association of MHC class-I alleles with particular outcomes of HIV-1 and SIV infections, ${ }^{19}$ and it is hypothesized that this is related to the efficiency of virus-specific CTL responses. The CD8+T cell response to HIV-1 is unique because in some cases virus-specific cytolysis is detectable in PBMC in the absence of in vitro stimulation, ${ }^{20,21} \mathrm{a}$ phenomenon that has not been frequently reported in other chronic viral infections. Nevertheless, despite evidence that HIV-1-specific $\mathrm{CD} 8+\mathrm{T}$ cells are abundant and capable of cytolytic function, they are unable to fully suppress virus replication in vivo resulting in the progressive depletion of CD4+T cells and, ultimately, death in untreated individuals. Quite perplexingly as well, in multiple studies the administration of exogenous CD8+T cells has failed to result in significant decreases in plasma HIV-1 RNA concentrations. ${ }^{22-25}$

\author{
Volume I Issue I - 2014 \\ Pamela J Skinner,' Elizabeth Connick ${ }^{2}$ \\ 'Department of Veterinary and Biomedical Sciences, University \\ of Minnesota, USA \\ ${ }^{2}$ Department of Medicine, University of Colorado Anschutz \\ Medical Campus, USA
}

Correspondence: Pamela J Skinner, University of Minnesota, 197I Commonwealth Ave, Saint Paul, MN, USA, Tel 612-6242644,Email Skinn002@umn.edu

Received: April 17, 2014 | Published: April 24, 2014

Furthermore, increases in HIV-specific CTL through therapeutic vaccination or treatment interruption have resulted in little or no enhancement of virologic control. ${ }^{26-29}$

HIV-1 evades HIV-1-specific CD8+T cells through multiple mechanisms, which likely contribute to the inability of HIV-1-specific CTL to fully control HIV-1 replication. First, HIV-1 latently infected cells do not express viral proteins, which are essential to CD8+T cell recognition. Second, HIV-1 frequently mutates to evade CD8+T cell responses. ${ }^{19}$ Third, HIV-specific CD8+T cells fail to accumulate in lymphoid B cell follicles and are not able to effectively clear the follicular reservoir of HIV-1-producing cells ${ }^{3}$ (Figure 1). This might explain why individuals with functional HIV-specific CD8+T cells fail to fully suppress HIV-1 replication, and why infused exogenous $\mathrm{CD} 8+\mathrm{T}$ cells or augmented endogenous CTL responses failed to significantly impact viral control.

Future HIV-1 cure and vaccine strategies must address not only the latent reservoir of HIV-1 infected cells and HIV-specific CD8+T cell escape mutations, but also the reservoir of HIV-1 producing cells within B cell follicles. Strategies to induce follicular lysis, such as through administration of the B-cell depleting monoclonal antibody rituximab, might temporarily allow virus-specific CTL to access the follicles. However, this would profoundly impair humoral immunity, and furthermore, the effects would only be transient. Once the B cell population recovered and follicles were reconstituted, HIV-1 replication most likely would resume at that site. Perhaps a better approach to eradicate the reservoir of HIV-1 within follicles, and one that we are presently developing, ${ }^{30}$ is to engineer functional HIV-1specific CD8+T cells to express chemokine receptors, such as the follicular homing molecule CXCR5, to allow virus-specific CTL to access the follicular compartment and clear the reservoir of HIV-1 producing cells within follicles. This approach could be applied by itself or in conjunction with other approaches such as one to stimulate latently infected cells to express HIV-1. Additionally, this approach could be implemented using CD8+T cells directed against HIV-1 neoepitopes, ${ }^{19}$ in cases where HIV-specific CD8+T cell escape mutations are an issue. Ultimately, therapeutic approaches that enable HIVspecific $\mathrm{CD} 8+\mathrm{T}$ cells to enter lymphoid B cell follicles and clear HIV1 producing cells may lead to a functional cure for HIV-1. 

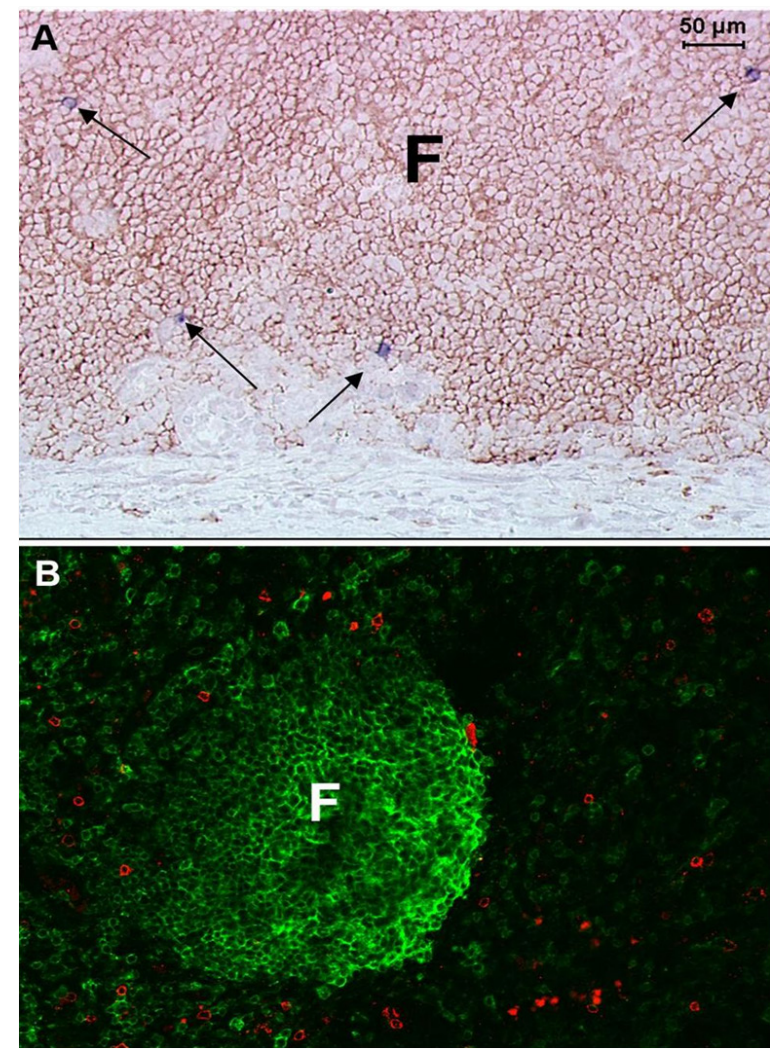

Figure 1 In lymph nodes from untreated HIV-1-infected individuals, virus replication is largely concentrated inside B cell follicles, whereas HIV-1-specific $\mathrm{CD} 8+\mathrm{T}$ cells fail to accumulate at those sites. A: HIV-1 RNA+ cells identified by in situ hybridization (blue-black staining cells indicated by arrows) are located primarily inside of a B cell follicle, defined morphologically by staining with anti-CD20 antibodies (brown); B: HIV-1-specific CD8+T cells labeled in situ with HLA-tetramers (red) are concentrated primarily outside of a B cell follicle, defined by anti-CD20 antibodies (green).

\section{Acknowledgments}

This work was supported by Public Health Services Grant R01AI096966 from the National Institutes of Health.

\section{Conflict of interest}

Authors declares that there is no conflicts of interest.

\section{References}

1. Embretson J, Zupancic M, Ribas JL, et al. Massive covert infection of helper T lymphocytes and macrophages by HIV during the incubation period of AIDS. Nature. 1993;362(6418):359-362.

2. Pantaleo G, Graziosi C, Demarest JF, et al. HIV infection is active and progressive in lymphoid tissue during the clinically latent stage of disease. Nature. 1993;362(6418):355-358.

3. Connick E, Mattila T, Folkvord JM, et al. CTL fail to accumulate at sites of HIV-1 replication in lymphoid tissue. J Immunol 2007;178(11):69756983.

4. Folkvord JM, Armon C, Connick E. Lymphoid follicles are sites of heightened human immunodeficiency virus type 1 (HIV-1) replication and reduced antiretroviral effector mechanisms. AIDS Res Hum Retroviruses. 2005;21(5):363-370.

5. Biberfeld P, Chayt KJ, Marselle LM, et al. HTLV-III expression in infected lymph nodes and relevance to pathogenesis of lymphadenopathy. Am J Pathol. 1986;125(3):436-442.
6. Hufert FT, van Lunzen J, Janossy G, et al. Germinal centre CD4+ T cells are an important site of HIV replication in vivo. AIDS. 199711(7):849857.

7. Mehandru S, Poles MA, Tenner-Racz K, et al. Primary HIV-1 infection is associated with preferential depletion of CD4+ T lymphocytes from effector sites in the gastrointestinal tract. J Exp Med. 2004;200(6):761770 .

8. Orenstein JM. HIV expression in surgical specimens. AIDS Res Hum Retroviruses. 2008;24(7):947-955.

9. Tenner-Racz K, Racz P. Follicular dendritic cells initiate and maintain infection of the germinal centers by human immunodeficiency virus. Curr Top Microbiol Immunol. 1995;201:141-159.

10. Tenner Racz K, Stellbrink HJ, van Lunzen J, et al. The unenlarged lymph nodes of HIV-1-infected, asymptomatic patients with high CD4 T cell counts are sites for virus replication and CD4 T cell proliferation. the impact of highly active antiretroviral therapy. J Exp Med. 1998;187(6):949-959.

11. Heath SL, Tew JG, Tew JG, et al. Follicular dendritic cells and human immunodeficiency virus infectivity. Nature 1995;377(6551):740-744.

12. Borrow P, Lewicki H, Hahn BH, et al. Virus-specific CD8+ cytotoxic T-lymphocyte activity associated with control of viremia in primary human immunodeficiency virus type 1 infection. $J$ Virol. 1994;68(9):6103-6110.

13. Koup RA, Safrit JT, Cao Y, et al. Temporal association of cellular immune responses with the initial control of viremia in primary human immunodeficiency virus type 1 syndrome. J Virol. 1994;68(7):4650-4655. 
14. Connick E, Marr DG, Zhang XQ, et al. HIV-specific cellular and humoral immune responses in primary HIV infection. AIDS Res Hum Retroviruses 1996;12(12):1129-1140.

15. Schmitz JE, Kuroda MJ, Santra S, et al. Control of viremia in simian immunodeficiency virus infection by $\mathrm{CD} 8+$ lymphocytes. Science. 1999;283(5403):857-860.

16. Jin X, Bauer DE, Tuttleton SE, et al. Dramatic rise in plasma viremia after CD8(+) T cell depletion in simian immunodeficiency virus-infected macaques. J Exp Med. 1999;189(6):991-998.

17. Riou C, Burgers WA, Mlisana K, et al. Differential impact of magnitude, polyfunctional capacity, and specificity of HIV-specific CD8+ T cell responses on HIV set point. J Virol. 2014;88(3):1819-1824.

18. Betts MR, Nason MC, West SM, et al. HIV nonprogressors preferentially maintain highly functional HIV-specific CD $8+\mathrm{T}$ cells. Blood. 2006;107(12):4781-4789.

19. Goulder PJ, Watkins DI. Impact of MHC class I diversity on immune control of immunodeficiency virus replication. Nat Rev Immunol. 2008;8(8):619-630.

20. Walker BD, Chakrabarti S, Moss B, et al. HIV-specific cytotoxic T lymphocytes in seropositive individuals. Nature. 1987;328(6128):345348.

21. Ferbas J, Kaplan AH, Hausner MA, et al. Virus burden in long-term survivors of human immunodeficiency virus (HIV) infection is a determinant of anti-HIV CD8+ lymphocyte activity. $J$ Infect Dis. 1995;172(2):329-339.

22. Koenig S, Conley AJ, Brewah YA, et al. Transfer of HIV-1-specific cytotoxic $\mathrm{T}$ lymphocytes to an AIDS patient leads to selection for mutant HIV variants and subsequent disease progression. Nat Med. 1995;1(4):330-336.

23. Lieberman J, Skolnik PR, Parkerson GR, et al. Safety of autologous, ex vivo-expanded human immunodeficiency virus (HIV)-specific cytotoxic T-lymphocyte infusion in HIV-infected patients. Blood. 1997;90(6):21962206
24. Mitsuyasu RT, Anton PA, Deeks SG, et al. Prolonged survival and tissue trafficking following adoptive transfer of CD4zeta gene-modified autologous $\mathrm{CD} 4(+)$ and $\mathrm{CD} 8(+) \mathrm{T}$ cells in human immunodeficiency virus-infected subjects. Blood. 2000;96(3):785-793.

25. Brodie SJ, Lewinsohn DA, Patterson BK, et al. In vivo migration and function of transferred HIV-1-specific cytotoxic T cells. Nat Med. 1999;5(1):34-41.

26. Autran B, Murphy RL, Costagliola D, et al. Greater viral rebound and reduced time to resume antiretroviral therapy after therapeutic immunization with the ALVAC-HIV vaccine (vCP1452). AIDS 2008;22(11):1313-1322.

27. Kinloch de Loes S, Hoen B, Smith DE, et al. Impact of therapeutic immunization on HIV-1 viremia after discontinuation of antiretroviral therapy initiated during acute infection. J Infect Dis. 2005;192(4):607617.

28. Goujard C, Marcellin F, Hendel-Chavez H, et al. Interruption of antiretroviral therapy initiated during primary HIV-1 infection: Impact of a therapeutic vaccination strategy combined with interleukin (IL)-2 compared with IL-2 alone in the ANRS 095 randomized study. AIDS Res Hum Retroviruses. 2007;23(9):1105-1113.

29. Markowitz M, Jin X, Hurley A, et al. Discontinuation of antiretroviral therapy commenced early during the course of human immunodeficiency virus type 1 infection, with or without adjunctive vaccination. $J$ Infect Dis. 2002;186(5):634-643.

30. Haran P, Rakasz E, Kaushik K, et al. Transduction of primary rhesus macaque CD8 T cells with the B cell homing molecule CXCR5. Keystone Symposia: HIV Pathogenesis, Banff Canada. 2014. 